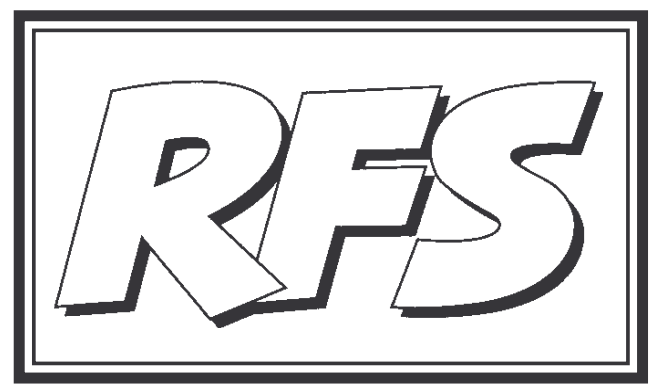

Revista de Fomento Social, 61 (2006), 561-586

\title{
Las Agencias de Desarrollo Regional (ADR) en el siglo XXI: hacia una reconfiguración de su marco de actuación operativa
}

(Palabras clave: Desarkollo Regional, Agencia de Desarkollo Regional (ADR), ESPAÑA.

Keys WORDS: Regional DeVElopment, Regional DeVelopment Agencies, SPAin)

${ }^{1}$ Agencia de Innovación y Desarrollo de Andalucía IDEA, Gerencia Provincial de Córdoba. 


\section{Justificación introductoria}

En un trabajo anterior publicado en esta misma Revista de Fomento Soéial intentamos realizar una aproximación, si se quiere somera, a lo que se deno minaba el 'modelo español de Agencias de Desarrollo Regional (ADR)'.

Sin ánimo de repetirnos, permítasenos, al menos, que destaquemos algunas consideraciones que consideramos necesarias:

a) En primer lugar, hemos de destacar que el necesario punto de partida del modelo analizado nacía del mismo 'Desarrollo Regional' ${ }^{3}$ como precipitante de todo el sistema de ADR, tanto en su escala europea como, a un nivel inferior, española.

b) En segundo lugar, y previa aproximación histórica al contexto español de referencia, nos permitíamos efectuar tanto una delimitación del modelo como una caracterización tipológica del mismo ${ }^{4}$ para acabar efectuando valoraciones de índole comparativa.

c) Como colofón de todo lo anterior, finalizábamos realizando algunas conclusiones (la falta de concreción conceptual que envuelve todo el tema analizado -'desarrollo regional' incluido-; la variedad y pluralidad detectadas; $y$, como resultado de todo lo anterior, la complejidad exis tente al respecto cuando se intenta efectuar comparaciones entre ADR

2 F. A. Aanguren Baena (2005).A este artículo, y a modo de complemento necesario del presente, hemos de remitir a todos los interesados en el tema más amplio de las ADR y su vinculación con el Desarrollo Regional.

${ }^{3}$ Cuyo concepto, aunque desbordaba cualquier intento de limitación y concreción, -confi gurándose como algo complejo, evolutivo, plural y diverso-, bien podíamos caracterizar como todo aquel proceso que genera cambios sociales y económicos beneficiosos para una región, concibiéndose como un todo holístico en el que los distintos actores de dicha región participan en la misma definición, decisión e implementación de dicho desarrollo, desde un nivel estratégico hasta uno más operativo

${ }^{4}$ Lo que, entre otros aspectos no menos importantes, nos permitía ensayar la siguiente de finición de ADR: toda organización que, desde una óptica eminentemente territorial y bajo las directrices de política económica emanadas de la autoridad pública correspondiente, realiza actuaciones de desarrollo, fomento y promoción empresariales, contando para ello con una amplia variedad de instrumentos, financieros o no, y de una regulación 'ad hoc' que le confiere ciertas características diferenciadoras de la simple Administración Pública. Siempre, eso sí, bajo una perspectiva estratégica centrada, no tanto en el mismo sujeto a auxiliar, sino en el desarrollo regional del conjunto territorial en el que se ubica la ADR que le confiere su misma razón de ser. 
o, lo que es más importante, realizar valoraciones del mismo modelo que se intenta analizar).

No obstante todo lo referido, en la parte final del trabajo comentado se dejaban apuntadas algunas 'carencias' y 'debilidades' del modelo español de ADR que, aparte lo referido, apuntaban a consideraciones tales como la creciente burocratización de las entidades, el riesgo acentuado de dirigismo político de las mismas, la falta d'Đenchmarking', las disfunciones con y entre otros organismos públicos con similares competencias, el excesivo acento en las ayudas financieras y en las fórmulas tradicionales (subvenciones) como instrumento de ayuda prioritario de actuación, la rigidez y obsolescencia en su estructura y funcionamiento, ... la pérdida creciente, en fin, de la credi bilidad del modelo español de ADR que conducía irremediablemente a una continuada pérdida de protagonismo de estas entidades, amén de un claro detrimento en su autonomía de funcionamiento y operatividad.

El presente trabajo, evidentemente, ha de tomar como necesario punto de arranque dichas consideraciones para, a la luz del nuevo contexto regional de actuación pública que se vislumbra en los comienzos de esta nueva cen turia, intentar presentar algunas reflexiones que, de alguna manera, ayuden a paliar las carencias y debilidades detectadas en el modelo de ADR y, a partir de éstas, consolidarlo a través del refuerzo de sus aspectos positivos (que también los hay) reduciendo en la mayoría de lo posible los negativos existentes.

En consonancia con nuestras pretensiones, y tomando como guía la metodología ya ensayada con anterioridad, empezaremos nuestro trabajo analizando sucintamente el nuevo marco en el que se desenvuelve eso que denominamos 'el desarrollo regional' para, a partir del mismo y enfocando también como necesario catalizador el contexto europeo de actuación operativa y normativa, efectuar algunas reflexiones, a modo de apuntes, de lo que a nuestro juicio han de ser las directrices de actuación que deben dirigir el modelo español de ADR en los próximos años. Sintetizando, lo que pretendemos en las próximas páginas es (re)configurar el marco teórico de actuación que ha de caracterizar el citado modelo para que éste pueda cumplir positivamente con los requisitos de eficiencia y eficacia que han de dirigir toda actuación, la pública incluida. Lógicamente, y dada la amplitud, variedad y complejidad del tema a analizar, cuando se decida ejemplificar acudiremos a la realidad más cercana a nosotros (geográfica y operativamen te) que no es otra que la Agencia de Innovación y Desarrollo de Andalucía IDEA (antiguo Instituto de Fomento de Andalucía, IFA). 
Tal vez se nos pueda acusar de pretender ser demasiados ambiciosos en la tarea que acometemos (jnada menos que intentar plasmar las directrices de índole teórica que han de marcar en los próximos años el modelo español de ADR!), crítica que asumimos, pero en nuestro intento hacemos nuestras las reflexiones de Pigou $^{5}$ cuando consideraba que el argumento económico está continuamente desempeñando un papel cada vez más importante en los debates políticos en los que, generalmente, estos acostumbran a decir primero lo que quieren hacer y buscan después los argumentos a favor de ello. Ya que no somos políticos y sí economistas, nuestra intención (y más en un tema como el que aquí tratamos, tan sujeto a las discusiones y directrices de lo político) ha de ser la contraria: buscar y ofrecer dichos argumentos teóricos para que, si es posible, después otros los lleven a la práctica haciéndolos suyos.

\section{Las nuevas estrategias del desarrollo regional}

En el referido trabajo anterior ${ }^{6}$, se dejaba reflejado que la perspectiva endógena (o enfoque de potencial endógeno) se configura como "la nueva teoría del desarrollo regional" 7. Bajo este "nuevo modelo"8 empiezan a co brar protagonismo los procesos definidos de 'abajo-arriba' a partir de la confluencia de tres variables interrelacionadas: la territorial (el ámbito de lo regional), la institucional (lo público) y la empresarial (lo privado). No obstante esto, también quedaba señalada la falta de concreción conceptual en la que parecía moverse el mismo 'desarrollo regional', lo que a juicio de algunos autores se plasma en una evidente "falta de postulados teóricos orientativos" a este respecto.

En este contexto, no es de extrañar que, aún reconociendo la primacía de lo endógeno, se empiece también a considerar "la necesidad de diseñar nuevas estrategias para hacer frente a los crecientes retos" 10 . Aunque, no sea éste el lugar idóneo para efectuar el state of the art' por lo que a las nuevas

5 A.C. Pigou (1944), pp. 9-30.

6 F. A. Aranguren Baena (2005), Pp. 591-594.

7 D. Coronado Guerrero (1997), pp. 50-53.

8 E. Furió Blasco (1996).

9 J. L. Russo (1997), p. 113.

10 E. I. Palavicini Corona (2000), p. 215. 
teorías de desarrollo regional se refieren, sí hemos de señalar que, como no podía ser de otra manera, no existe unanimidad a este respecto y cada autor o escuela doctrinal parece tener su propia opinión y punto de vista ${ }^{11}$. No obstante, y pese a esto, sí parece existir cierta coincidencia en cuáles han de ser los objetivos de estas nuevas estrategias. Estos bien puede resumirse en los siguientes ${ }^{12}$ : aumentar el potencial regional para responder activamente a problemas de desempleo y exclusión social, búsqueda de un desarrollo regional sostenible, impulso a la creación de empleos y la existencia y propa gación de una cultura de emprendedores, estímulo de redes de cooperación con la meta de reconciliar economía y sociedad, y establecer el análisis y la evaluación constante de las políticas de desarrollo a implementar.

Si bien puede existir cierta unanimidad en los objetivos pretendidos, tal y como hemos apuntado no parece ser este el caso cuando nos situamos en el lado de las estrategias concretas de desarrollo regional a implementar. Debido a esto, cada vez parece cobrar mayor importancia y protagonismo las vías eclécticas que, en el marco del enfoque endógeno comentado, tienden a buscar aproximaciones multidisciplinares y multidimensionales al problema planteado. Basándose en este método, se pretende desarrollar un marco con ceptual del desarrollo regional que permita un intento de caracterización del nuevo paradigma existente en el ámbito del desarrollo regional. Esta nueva aproximación, toma como punto de arranque la misma filosofa del enfoque endógeno y su conocida trilog ía (lo territorial, lo p úblico y lo privado). A partir de aquí, y ésta es la novedad, aplica una vía 'inter-organizacional' en la que a partir de la promoción política y, sobre todo, la implementación de una organización intermedia es posible conectar con los verdaderos obje tivos de esta estrategia en aras de la consecución de un exitoso desarrollo regional: los actores empresariales privados.

Bajo este nivel de an álisis, la parte m ás delicada y sensible de toda la política de desarrollo regional a llevar a cabo es la de la implementacŏn de dicha organización intermedia. Para el buen fin dise ñado, resulta del todo punto imprescindible definir con la mayor claridad posible tres dimensiones

11 E. MonCAYo JimÉnEz (2002) ofrece una sistematización de las diferentes perspectivas teóricas que están confluyendo en lo que el propio autor denomina como "nuevo paradigma del desarrollo territorial"en el que emerge el "estado de trabajo schumpeteriano".

12 J. L. R usso (1997), pp. 218-220. Este autor basa su presentación en B. HugonNiER (1999) y, debido a esto, bien podemos considerar dichos objetivos como perfectamente asumidos y respaldados por la Organización para la Cooperación y Desarrollo Económicos (OCDE). 
esenciales de la misma: sus recursos, sus competencias y sus estrategias o fines. Sólo así sería posible que esta entidad organizacional pudiera llegar a conseguir que los actores privados interactuaran con los podeń́dslixos bajo el marco de una reglas de juego perfectamente definidas y delimitadas.

Evidentemente, y dada la estrategia descrita, las caractísticas, funciones, presupuestos, descripción y naturaleza de esta implementacôn organizacional queda al arbitrio de dichos poderes públicos (sin olvidar, bajo el 'parte nariado' aludido, las consideraciones y demandas del sector privado). No obstante, para los defensores de esta nueva aproximacîn al marco analtico del desarrollo regional, estas organizaciones han de identificarse en todo caso con las conocidas como Agencias de Desarrollo Regional (ADR). Hasta tal punto que, a la luz de esta aproximacón, es posible reinterpretar incluso las bases del conjunto de la poltica regional en Europa Occidental En otras palabras, se pasa del anterior paradigma de las ṕtodas regionales 'top-down' al más actual, reciente y novedoso de las Agencias de Desarrollo Regional. Y es que éste último permitiría compatibilizar las iniciativas de crecimiento endógeno en el marco del desarrollo regional con la tendencia general hacia la implementación 'por proximidad' al territorio donde se pretende actuar para corregir los desequilibrios existentes. En resumen, y citandøin extenso las palabras de Halkier ${ }^{13}$, uno de los principales defensores de esta nueva vía de aproximación inter-organizacional:
"En otras palabras, una transición de un paradigma a otro ciertamente establecería una diferencia en la política regional, y en las situaciones donde ambos paradigmas coexistieran, esto podría ser el resultado de actores nacionales y regionales adoptando posiciones estratégicas que podrían ir desde la cooperación a la competenciaEn este contexto apenas sería sorprendente que las dos aproximaciones hayan sido vistas como paradigmas alternativos en la política regional, como dos formas de intervención pública en las que ambos están presentes para corregir los desequilibrios espaciales pero que implican actores diferentes, niveles de operatividad diferentes, supuestos subyacentes diferentes, estrategias diferentes, y diferentes formas de interacción entre las entidades públicas y privadas, -y además y últimamente consecuencias diferentes para la política de la política regional".

13 H. HALKIER (2000), p. 45: "A transition from one paradigma to another would in other words certainly make a difference to regional policy, and in situations where the two paradigms coexist, cohabitation may result in national and regional actors adopting positioning strate gies ranging from cooperation to competion. On this background it is hardly surprising that the two approaches have been seen as alternative paradigms in regional policy, two forms of public intervention that are both concerned with remedying spatial inequalities but which involve different actors, different spatial levels of operation, different underlying assump tions, different strategies, and different forms of interaction between public and private bodies-and thus ultimately different consequences for the politics of regional policy". 
Evidentemente, para que este nuevo paradigma pueda resultar plenamente satisfactorio para todos los actores implicados y pueda llegar a alcanzar los objetivos marcados en la nueva estrategia de desarrollo regional, resulta ineludible que la configuración de las mencionadas ADR se adapte al mismo. Dicho de otra manera, si se ha de reconfigurar la estrategia de lo regional en su conjunto, necesariamente tambén ha de reconfigurarse la caracterizaćin operativa del actual modelo de ADR. Para esto, y antes de descender a un nivel más desagregado de análisis, resulta necesario que nos detengamos, siquiera con brevedad, en el contexto europeo para conocer las posibles novedades a este respecto.

\section{La nueva Política Regional Europea: la Estrategia de Lisboa}

Siguiendo la metodología ya ensayada en nuestra anterior investigacón ${ }^{14}$ que nos llevaba a trav és de un proceso de inferencia desde lo general (el mismo concepto de desarrollo regional) a lo $\mathrm{m}$ ás particular (el modelo español de ADR), consideramos el contexto europeo como catalizador de la reconfiguración a realizar en el marco operativo de las ADR españolas. Y bajo este considerando, cuando nos referimos al contexto europeo, ineludi blemente hemos de referirnos a la nueva Política Regional Europea y, como precipitante de la misma, la conocida como Estrategia de Lisboa.

Los días 23 y 24 de marzo de 2000, el Consejo Europeo extraordinario de Lisboa se comprometió (en lo que se conoce como la Estrategia de Lisboa) hacer de la economía europea la más dinámica y competitiva del mundo en el plazo de 10 años. En este sentido, los objetivos globales de dicha estrategia abarcaban diferentes y relevantes ámbitos a alcanzar en el umbral de 2010 que, someramente, podemos sintetizar en dos grandes apartados: a) un im pulso al crecimiento económico y la productividad, así como a la creación de empleo de calidad; y b) una mejora de la competitividad empresarial para crear un clima favorable al espíritu emprendedor, a través del desarrollo de políticas relacionadas con la capacidad innovadora y tecógilca, la educacón y formación (sociedad del conocimiento), y la internacionalización.

En 2005 se llevó a cabo un balance intermedio de dicha estrategia. Dados los escasos y desiguales resultados obtenidos hasta dicho momento, fue necesario proponer un nuevo proceso de coordinaón junto con una concen tración de los esfuerzos comprometidos. De acuerdo con esto, la Comisión

14 F. A. Aranguren Baena (2005), pp. 594-599. 
Europea ${ }^{15}$ decidió no centrar la atención en objetivos concretos sino en las acciones a llevar a cabo que, enístesis, podemos concretar en las siguientes: más crecimiento, más puestos de trabajo y de mejor calidad, y una mejor gobernanza. Entendida esta última como un nuevo estilo de relaci ón entre las esferas pública y privada, se vincula a un reparto de responsabilidades más claro y eficaz, intentando reducir la excesiva burocraciaía, entre otras, una simplificación administrativa.

Tomando como base esta Estrategia de Lisboa, la Uróin Europea presenta unas propuestas concretas sobre el futuro de su política regional posterior a 2006 y elabora sus directrices estratégicas para el período $2007-2013^{16}$. Al no ser éste el lugar idóneo para ello, no profundizaremos en este apartado, aunque sí nos gustaría resaltar que la cohesión territorial y la corrección de los desequilibrios entre las regiones europeas sigue siendo pilar fundamental en la política de cohesión europea. Así, uno de los objetivos expl ícitos de las nuevas directrices estratégicas es la garantía de una mejor integraci ón de la cohesión a nivel regional, lo que significa un dălogo reforzado entre la Comisión, los Estados miembros y las distintas regiones. No s ólo esto. Las directrices estratégicas comunitarias se concentran en torno a tres priorida des: la mejora del atractivo de las regiones y de las ciudades de los Estados miembros para la inversín y el empleo; el apoyo a la innovacôn, el fomento del espíritu empresarial y el crecimiento de la economía del conocimiento; y la creación de más empleos y de mejor calidad.

De acuerdo con lo anterior, y para conseguir los objetivos y fines propuestos, la Comisión Europea propone tener en cuenta especialmente la dimensi ón territorial de la pol ítica de cohesi ón, de tal modo que la pol ítica regional europea se adapte a las necesidades y a las caracter ísticas específicas de los territorios en función de los problemas u oportunidades que ofrezca su situación geográfica y socioeconómica.

Lógicamente, es en este nuevo contexto donde deben definirse tanto el papel como las funciones y los objetivos de las ADR como organizaciones intermedias a implementar en su tarea de servir de cauce privilegiado donde interactúen las estrategias y poíticas macro (elámbito de lo público) con las necesidades y demandas micro (el ámbito de la empresa privada, la PYME sobre todo).

15 COM (2005) 24 final, de 2 de febrero de 2005.

16 COM (2005) 299 final, de 5 de julio de 2005. 


\section{Hacia una nueva caracterización de las Agencias de Desarrollo Regional}

Tal y como ha quedado explicitado en otras obras ${ }^{17}$, resulta complejo, cuando no imposible, establecer una caracterización unívoca de lo que es una Agencia de Desarrollo Regional. Empezando por su misma indefinición conceptual, en la que no parece existir mucha coincidencia entre las distintas definiciones ensayadas por los distintos autores. Nosotros, tal vez con un ánimo conciliador de todas las corrientes existentes, optamos por utilizar la definición recogida en la nota 4.

Salvando dichos problemas de caracterización conceptual, no parece difícil, en cambio, justificar, al menos teóricamente, la existencia de las ADR. Aunque, evidentemente, cada caso parece responder a unas causas determinadas en función del territorio (la región) donde operan específicamente, sí podemos explicitar que las Agencias de Desarrollo suelen surgir, tal y como se ha apuntado con anterioridad, como consecuencia tanto de la falta de validez de la política regional tradicional (el comentado paradigma 'top-down') para la resolución de los desequilibrios regionales existentes, como para hacer frente al nuevo enfoque endógeno del desarrollo regional. No obstante, y debido precisamente a esto, cuando se produce un punto de inflexión cualitativo en el marco teórico que soporta analíticamente la misma existencia de las ADR (un cambio de paradigma, en palabras de Halkier ${ }^{18}$ ), éstas, para su adaptación al nuevo modelo, deben ineludiblemente, a riesgo de su desaparición por incapacidad (en términos de eficacia y eficiencia) u obsolescencia (falta de respuesta a las necesidades de sus 'clientes': el sector empresarial privado) adaptarse a la nueva situación, a través de una reconfiguración de sus objetivos, instrumentos, recursos y funciones, de su marco de actuación operativa, en resumidas cuentas.

En las próximas páginas intentaremos plasmar algunas recomendaciones normativo-teóricas que deben actuar como guía para la nueva caracterización del modelo español de ADR para que éste pueda seguir operando con garantías de éxito en sus resultados. Para que no se nos acuse de prestar

17 H. Halkier Et Al. (1998), Eurada (1999a), H. Halkier (2000), F. A. Aranguren Baena (2005), entre otras.

18 H. HALKIER (2000), p. 42: "The paradigms should, however, not be seen as normative ideals, prescribing how policy ought to have been, but rather as empirical generalisations (...)"/"Los paradigmas, sin embargo, no han de ser vistos como ideales normativos, que prescriban ómo debe de ser la política, sino más bien como generalizaciones empíricas(...)”. 
demasiada atención al marco teórico de actuación olvidando la realidad empírica ('el caso práctico'), estas a modo de prescripciones toman como referencia inmediata un caso real: el de la Agencia de Innovación y Desarrollo de Andalucía IDEA, la ADR andaluza que, desde el 1 de enero de $2005{ }^{19}$, ha sustituido en su actuación al Instituto de Fomento de Andalucía (IFA). Así, a través del proceso seguido por ésta desde el desarrollo del Libro Blanco para un 'nuevo Instituto de Fomento de Andalucía'20 hasta su continuación inmediata, el 'Plan Estratégico 2005/2008' ${ }^{21}$ (el cual, a su vez, toma como referente el Plan de Innovación y Modernización de Andalucía ${ }^{22}$ ), será más fácil que pueda elevarse, a través de un proceso de inducción metodológica, a categoría o recomendación general (el conjunto del modelo español de ADR) determinadas actuaciones ya implementadas con carácter particular (la ADR andaluza) ${ }^{23}$.

\subsection{Los principios inspiradores}

Aparte el ya mencionado contexto de referencia (europeo, nacional y au tonómico) donde cada ADR opera geográfica y territorialmente y que bien podemos caracterizar como el ámbito de lo político-territoriałt que, de una manera u otra, las determina normativa, presupuestaria y funcionalmente,

19 BOJA n ${ }^{\circ} 255$, de 31 de diciembre de 2004 .

20 Instituto de Fomento DE Andalucia IFA (2004). Este Libro Blanco (sin publicar oficialmente) fue aprobado por el Consejo Rector del entonces IFA con fecha 26/10/2004 y, posteriormente, por el Consejo de Gobierno de la Junta de Andalucía de 09/11/2004.

21 Agencia de Innovacion y Desarrollo de Andalucia IDEA (2006).

22 Consejería De InNovacion, Ciencia y Empresa (2004).

23 Evidentemente, con esto no se quiera ver a la Agencia andaluza como 'modelo' necesario del nuevo marco de actuación operativa que recomendamos. Nuestra intención, repetimos, sólo pasa por plasmar una referencia práctica de lo que significa para una ADR el tener que plantearse un cambio en su mismo modelo configurativo y operativo. Entre otras cosas, dicho cambio -y sirva aquí como ejemplo el caso andaluz- se ve motivado por las mismas deficiencias del anterior modelo, las cuales ya fueron explicitadas en nuestro anterior trabajo, al cual hemos de remitir necesariamente.

24 Por ejemplificar, y en el caso de la ADR andaluza, el marco de referencia más inmediato (aparte el europeo y nacional, común a todas las Agencias de Desarrollo españolas) hay que situarlo en la puesta en marcha del proyecto de la Segunda Modernización de Andalucía y su correlato teórico inmediato: el mencionado Plan de Innovación y Modernización de Andalucía (2004). 
cada Agencia debe saber identificar los principios inspiradores que deben guiar su nuevo modelo de actuación, lo que no es sencillo, ya que "hay, por supuesto, muchos modelos existentes de agencia regional que pueden ser usados como guía de las estructuras y operaciones de una $A D R$ ”. ${ }^{25}$

En nuestro caso concreto, y aunque la misma EURADA ${ }^{26}$ (Asociación Europea de Agencias de Desarrollo Regional) ha establecido algunos criterios orientativos a este respecto, fruto de una metodología de índole comparativa entre sus miembros, como tal modelo o guía preferimos utilizar el ejemplo de la Agencia andaluza que, en síntesis, ha establecido como tales principios de actuación los siguientes:

a. la excelencia (en sus funciones);

b. el liderazgo (en la identificació n de los procesos de cambio regionales);

c. la reflexió n y el debate internos (aprender del pasado para afrontar el presente y pensar en el futuro);

d. la dirección y la gestión (derivadas de una planificación operativa y estratégica);

e. la ejecución competencial (en las políticas identificadas como de su ámbito competencial); y

f. el reforzamiento de su imagen pública (ante los actores públicos y priva dos que interactúan en la puesta en marcha de la política regional).

\subsection{El marco teórico de actuación operativa}

De acuerdo a sus principios inspiradores, el nuevo modelo de las ADR españolas debe construir y configurar un marco teórico de actuación que le sirva para definir su papel en la nueva situación donde han de operar las mismas para que, tomando como referente necesario el modelo tradicional de Agencia de Desarrollo Regional creado en la pasada centuria, afrontar los desafíos de "una forma distintiva de la política moderna que es funcional y territorialmente desagregada pero, sin embargo, se encuentra vinculada

25 P. ROBERTS (1999), p. 5: "There are, of course, many existing models of regional agency that can be used to guide the structure and operation of the RDAs"

26 Eurada (1999a). 
conjuntamente y con la sociedad a través de una red de relaciones interguber namentales e interorganizacionales" 27.

En otras palabras, y parafraseando lo señalado con anterioridad, las ADR han de diseñar su paradigma de actuación con la finalidad de focalizar sus aspectos esenciales. No obstante, no ha de perderse de vista que esta configuración teórica debe entenderse tanto como algo integral, que afecte y vincule a todas y cada una de las funciones, recursos y objetivos a desarrollar por las Agencias de Desarrollo Regional, como algo con vocación de finalidad última, cuyo resultado debe ser alcanzado tras la ejecución material de las medidas estratégicas establecidas a este respecto.

Aunque, evidentemente, no existe ningún modelo-tipo que sirva como orientación básica a este respecto ${ }^{28}$, algunos autores ${ }^{29}$ sugieren que, al menos, para su diseño se debe tener en cuenta los criterios organizativos, estratégicos y de implementación práctica, los cuales deben enfocarse bajo una visión integrada y holística.

De acuerdo con éstos, la configuración teórica de la ADR debería configt rarse bajo un doble pilar: su modelo de actuación (o el ámbito externo de su actuación) ysu modelo de organización (o el ámbito interno de su actuación). El primero, es el que debe establecer los elementos que configuren el proyecto de intervención pública de la ADR. Por tanto, debe ser equiparado con la esfera 'externa' de la entidad, en cuanto sirve para fijar la acción exterior de la misma y sus relaciones con los destinatarios de su actuación operativa; el segundo, que bien podemos equipararlo al ámbito de lo ‘interno', debe servir para establecer las bases de mejora y transformación del modelo para conseguir el mejor desempeño de sus funciones, por lo que debe centrarse en la definición de su funcionamiento interno.

27 C. ANSELl (2000), p. 303: "A distinctive form of modern polity that is functionally and territorialy disaggregated, but nevertheless linked together and linked to society through a web of interorganizational and intergovernmental relationships".

28 Al igual que para los principios inspiradores, en EURADA (1999a) se pueden encontrar ideas para la configuración del marco teórico de actuación de las ADRNo obstante, algunas de las mismas -dada la relativa antigüedad del documento de trabajo- se pueden considerar como ya superadas, y más que a una reconfiguración de un nuevo modelo de ADR deberíamos vincularlas a un modelo tradicional de Agencias de Desarrollo.

29 H. HALKIER ET AL. (1998). 


\subsubsection{Configuración externa del modelo}

Este componente debería recoger tanto la definición de los principios básicos de la institución (misión, valores,...), como de los objetivos y principios de actuación de la misma. No obstante, al centrarnos sobre el ámbito de proyección externa de la ADR, no debe nunca olvidarse que todo este diseño debe pivotar sobre los destinatarios finales (los 'clientes') de la actuación operativa de las Agencias donde estos interactúan: tanto los actores públicos (la administración, en su triple vertiente europea, nacional y autonómica), como los privados (las empresa30, -identificadas, las más de las veces, con las PYMES que operan en el ámbito territorial de referencia de cada ADR-, y los centros tecnológicos, centros de innovación, centros de emprendedores, etc.).

Evidentemente, y como se ha explicitado en numerosas ocasiones, dada la complejidad y diversidad del modelo español de ADR, resultaría pretencioso (y equivocado) por nuestra parte el que intentemos definir ' ad hoc' cada uno de estos propósitos básicos para cada una de las ADR españolas ${ }^{31}$. No obstante, y a modo de 'común denominador' del conjunto, sí podemos tomar como referencia válida los establecidos a estos respectos por la Agencia de Innovación y Desarrollo de Andalucía IDEA en su Plan Estratégico 2005/2008, el cual toma como referente inmediato el Plan de Innovación y Modernización de Andalucía. Así, ésta identifica como su Misión principal ${ }^{32}$ :

"contribuir al desarrollo económico y social de la región andaluza, ofreciendo los mejores servicios a las empresas andaluzas, a los emprendedores y a la propia Junta de Andalucía, fomentando el espíritu empresarial, la innovación, la cooperación en el sistema ciencia-tecnología-empresa y la competitividad de la estructura productiva andaluza".

Igualmente, para la ADR andaluza sus Objetivos y Principios de Actuación son los siguientes ${ }^{33}$ :

30 En EURADA (1999b) se pueden encontrar interesantes reflexiones sobre la necesaria relación entre las ADR y las empresas, y como ambas interactúan y se influyen mutuamente.

31 En otros modelos no parecen existir mayores problemas a este respecto, ya que estos prin cipios están establecidos normativamente. Este es el caso de las ADR inglesas, en los que claramente se delimitan "the purposes of the RDAs"en la cláusula 4 deRegional Development Agencies Bill (Bill 100 of 1997/98). A este respecto, véase J. Dyson ET AL. (1998),

32 Agencia de Innovación y Desarrollo de Andalucía IDEA (2006), p. 19.

33 Agencia de Innovación y Desarrollo de Andalucía IDEA (2006), p. 20 
I. Implantación de nuevos mé todos y modelos de gobernanza.

II. Apuesta por la Innovació n e Inteligencia Territorial.

III. Promoción del 'partenariado' Pú blico-Privado.

IV. Fomento del espí ritu empresarial e innovador.

V. Diseño e implantació n de servicios de apoyo de calidad.

VI. Dotación de capital para apoyo y financiació n de las empresas.

VII. Establecimiento de un entorno administrativo adecuado para la crea ción empresarial.

VIII. Promoción de redes de cooperación entre agentes del sistema innovación-ciencia-empresa.

IX. Incorporació n de criterios culturales medioambientales y de mejora de la calidad de vida.

X. Apuesta por el ' benchmarking'.

Dada la importancia de estos Objetivos y Principios de Actuación para el marco de actuación de la nueva ADR, consideramos pertinente intentar clarificar algunos de los conceptos más novedosos aquí introducidos. Para esto, y dado que los mismos han sido tomados del Plan Estratégico de la Agencia andaluza, tomamos como referencia el Plan de Innovación y Moder nización de Andalucía y el Libro Blanco del 'nuevo Instituto de Fomento de Andalucía' 34 antecedentes inmediatos del referido Plan Estratégico.

a. Gobernanza: buscando como finalidad última la reducción de la buro cracia y de los trámites puramente administrativos, este concepto ha de ser entendido como un nuevo estilo de dirección y gobierno que contrasta y difiere de los tradicionales de control jerárquico y que se centra en la interacción y cooperación entre la administración y los actores no gubernamentales. Mediante este nuevo principio de actuación, es posible el establecimiento de redes de decisión mixta entre lo público y lo privado, implicando a todos los participantes en la estrategia de desarrollo regional mediante la implan tación de un consenso sobre los objetivos a medio plazo, así como sobre los medios e instrumentos a utilizar.

34 Instituto de Fomento de AndaluCia IFA (2004), pp. 24-29. 
b. Innovación ${ }^{35}$ e inteligencia territorial: por innovación ha de entenderse la capacidad de las empresas para introducir en el mercado nuevos produc tos, servicios y/o procedimientos, sin operar necesariamente en sectores de alta tecnología. En este ámbito, tan importante como el suministrar infraestructuras de proximidad, es la puesta en marcha de dispositivos de acompañamiento y de cooperación entre los centros universitarios y las empresas, sin olvidar los mecanismos de ingeniería financiera adaptados a los proyectos innovadores ${ }^{36}$. Por el contrario, el concepto de inteligencia territorial ha de ser entendido como la facultad de anticiparse a los cambios productivos (industriales, pero no exclusivamente) que puedan afectar a los territorios concernidos y a su tejido productivo, a medio y largo plazo. Esta inteligencia debe permitir la explotación óptima de las informaciones sobre la dinámica empresarial (de índole tecnológica, sobre todo). Este principio de actuación permite, entre otros, caracterizar a los distintos sectores productivos, favorecer las actividades de integración de nuevas tecnologías, establecer un mapa de recursos de excelencia innovadora, .... adaptar, en fin, continuamente los instrumentos de la ADR a la cambiante realidad empresarial. Y es que aquí encontramoła emergencia de una nueva idea-fuerza que enlaza directamente con la estrategia schumpeteriana de

35 Respecto a la importancia de la innovación y el papel a jugar a este respecto por las ADR, véanse las reflexiones realizadas en el marco del $36^{\circ}$ Congreso deEteropean Regional Science Association, celebrado en agosto de 1996. En concreto, la ponencia deB. URUEÑa GuTIÉRREz ET AL., titulada Technological policies and regional development in Spain: the role of Regional Development Agencies/Políticas tecnológicas y desarrollo regional en España: el papel de las $A D R$.

36 Ingeniería financiera entendida como la entiende la Comisión Europea, de acuerdo al documento de la misma titulado Guide to financial engineering techniques used by the European Commission in the context of regional policy/Guía de técnicas de ingeniería financiera usadas por la CE en el contexto de la política regional (1994), pp. 2-3: "financial engineering is, at its most basic, the process of developing solutions to financial problemsThe financial engineering process is thus a classic research and development process, involving identification of an oppor tunity, the conception and development of a solution, and finally its implementation. Accordingly, new financial products, more appropriate for these requirements, need to be developed in the context of Community regional policy For these reasons new financial products may henceforth benefit from ERDF assistance" /"Ia ingeniería financiera es, básicamente, el proceso de buscar soluciones a problemas financieros. Éste es un proceso de búsqueda y desarrollo, que implica identificación de oportunidades, la concepci ón y desarrollo de soluciones, y, finalmente, su implementación. De acuerdo a esto, los nuevos productos financieros, $m$ ás apropiados para los nuevos requerimientos, necesitan ser desarrollados en el contexto de la pol ítica regional comunitaria. Por estas razones, los nuevos productos financieros pueden beneficiarse de la asistencia del FEDER ”. 
apoyo a la empresa, en general, y la PYME, en particular:"la importancia del empresariado y la innovación tecnológica" 37.

En este contexto, las Agencias de Desarrollo asumirían el papel de

"responsables del 'aprendizaje' de los agentes potenciales de innovación. La forma de integrar a las empresas en redes que favorezcan el conocimiento y el aprendizaje para la innovación y la competitividad, así como mostrar el camino para la adquisición de formación de personal, son sus principales responsabilidades (...). Se echa de menos en estas regiones una estrategia 'bottom-up' que favorezca el 'aprendizaje', aunque por supuesto acompañada de una oferta de iniciativas 'top-down', porque, como también se ha constatado, las ayudas a la $I+D$ tienen resultados positivos con carácter general en todas las zonas"38.

c. 'Partenariado' Público-Privado: este objetivo( "una de las intervenciones más usadas por las estructuras intermedias es el reforzamiento de la coopeóraci empresarial tanto a nivel regional como transnacional" ${ }^{39}$ ) intenta llevar a la práctica lazos estables de actuación conjunta con los prestadores privados de servicios de apoyo a las empresas, en especial para la provisión de in fraestructuras y servicios tecnológicos, movilizándose recursos del sector privado a través de una mayor implicación de éste a través de las cadenas de valor, los clusters y, sobre todo, los distritos industriales ${ }^{40}$.

d. Espíritu empresarial: bajo el objetivo de aumentar la creación neta de empresas con potencial real de crecimiento e innovación, se debe realizar un importante esfuerzo en la promoción de la cultura empresarial en todos los sectores implicados, prestándose especial atención a la formación a la gestión, tutoría, intermediación, etc. Y es que, a pesar de que en el nuevo contexto económico-social que se vislumbra, el empresario tiende a configurarse como el prototipo de actuación operativa caracterizándose por su creatividad, competitividad e innovación ${ }^{41}$, también podría afirmarse que, a la par,

37 C. Ansell (2000), p. 314.

38 D. Coronado Guerrero et al. (1999), p. 115.

39 EURADA (1999b), p. 12: "One of the increasingly useful interventions of the intermediary structures is the reinforcement of business cooperation both regional level and a transnational level”.

40 Para una caracterización adecuada del concepto de distrito industrial, véaseV. SOLER I MARCO (2000).

41 Para una reflexión sobre el nuevo papel del empresario en el siglo XXI, véaßeFonTELA MonTES (2000). 
"la figura del empresario y la función empresarial siguen impregnadas de una fuerte dosis de desconocimiento científico, lo cual hace que las teorías de desarrollo regional se encuentren a menudo con un obstáculo insalvable para su aplicación empírica, espe cialmente cuando se trata de territorios con escasa cultura o espíritu de empresa". ${ }^{42}$

f. Servicios de apoyo de calidad: bajo el objetivo de complementar las actuaciones de corte más tradicional (incentivos financieros), este principio ha de concebirse en términos de compatibilidad con la iniciativa privada, sin ánimo de sustituirla, actuando a través de una amplia categoría de intangibles $^{43}$ : información, conocimiento, tutorización, asesoramiento, asistencia técnica,.... Bajo este nuevo concepto de actuación operativa, la tradicional (y, a veces, recurrente) intervención en empresas en crisis sólo puede concebirse con carácter excepcional y siempre por la importancia que pueda tener determinado sector o territorio.

g. Disponibilidad de capitales: bien conocida y abundantemente tratada por la literatura específica ha sido la dificultad tradicional de acceso al mer cado de capitales que presentan las empresas, las PYMES sobre todo, por lo que no es necesario insistir demasiado ponderando la importancia de este aspecto. Este objetivo promueve la oferta de todas las fórmulas de ingeniería financiera existentes (capital riesgo y garantía, incluidos) que permita a los empresarios tener acceso a los recursos necesarios para su desarrollo y/o innovación. Privilegiando la fórmula del 'paquete de actuaciones' así como los nuevos servicios integrados de financiación, este principio de actuación ha de complementarse con un servicio adecuado de asesoramiento y seguimiento. Bajo la filosofía de que cada tipo de financiación debe adaptarse a la situación concreta empresarial demandante de ayuda ('un traje a medida'), la ADR debe intentar que cada empresa esté perfectamente informada de la oferta exis tente de recursos financieros, así como los requerimientos y prescripciones exigidos. Como vemos, es necesaria una nueva aproximación al problema debido a que "la cuestión que se nos plantea precisamente en el terreno de las políticas empresariales es que tal vez haya que cambiar gran parte del diseño de la política de apoyo empresarial, especialmente a las PYMES (...)."44

h. Entorno administrativo adecuado: la nueva ADR ha de tener claro que, conforme a las directrices de la nueva política regional europea, han de

\footnotetext{
42 J. Guzmán Cuevas Et AL. (2001), p. 151.

43 Eurada (1999b): p. 7.

$44 M^{\mathrm{a}}$.R. Toribio Muñoz ET AL. (2001), p. 203.
} 
simplificarse al máximo los procesos administrativos a cumplimentar por los distintos actores privados, objeto último de la actuación operativa de la Agencia de Desarrollo. En este sentido, se ha de promover la creación de una relación interactiva entre las partes, a través de instrumentos telemáticos e informáticos.

i. Calidad de las redes: bajo el principio de que las redes empresariales son un factor decisivo en la competitividad regional, las nuevas ADR deben jugar un papel de precipitantes en la creación de las mismas. Este esfuerzo debe complementarse con operaciones de 'marketing' territorial que pi votaría sobre las excelencias del ámbito regional de actuación operativa, concentrándose la información en determinados sectores o empresas líderes en determinado segmentos de mercado.

j. Cultura, medioambiente y calidad de vida: cada vez más, a condiciones empresariales similares, los factores determinantes de atracción inversora y empresarial son los vinculados a la calidad de vida, así como a las redes de contactos formales e informales, financieros y de asesoramiento que la región pueda ofrecer. Igualmente, la cultura y los aspectos medioambientales empiezan a configurarse como nuevas opciones de mercado para la creación empresarial, mereciendo una atención y dedicación crecientes.

k. 'Benchmarking': por tal ha de entenderse las periódicas comparaciones a efectuar entre las distintas Agencias de Desarrollo y/o entre éstas y otros organismos que realicen actividades en campos similares de actuación. Sólo a través de este proceso, es posible incorporar mejoras continuas en las distintas funciones o metodologías a implementar, con el objetivo último de llegar a beneficiarse de las 'mejores prácticas' en el sector.

\subsubsection{Configuración interna del modelo}

En paralelo a la esfera externa, las ADR deben saber configurar los objetivos y principios básicos de actuación de su esfera interna: visión, valores inter nos, organigrama, funciones, procesos, instrumentos, recursos,... Si el tratar el ámbito externo del nuevo modelo a diseñar resultaba complicada, cuando no imposible, las generalizaciones entre distintas ADR, mucho más difícil resulta el tratar cuestiones de índole interna, ya que aquí el peso e influencia de la intrahistoria de cada una de ellas (a modo de su 'idiosincracia'), resulta fundamental para el establecimiento de unos u otros criterios. No obstante, y aunque algunos trabajos dan orientaciones generales sobre la organización 
interna de las $\mathrm{ADR}^{5}$, al igual que en el caso precedente preferimos tomar como referencia los establecidos por la ADR andaluza ya que, a partir de la misma, puede resultar más inteligible el ejercicio teórico a desarrollar.

De acuerdo a ésta, los principios y objetivos que rigen su modelo de or ganización interna son los siguientes ${ }^{46}$ :

i. Enfoque de la actividad a la satisfacció n de los clientes.

ii. Búsqueda de la excelencia en la prestació n de servicios.

iii. Fomento del conocimiento y la mejora profesional.

iv. Apuesta por la creatividad y la innovación.

v. Favorecer la transmisión de valores éticos.

vi. Implantación de un modelo de organizació transversal y sus procesos.

Evidentemente, dentro de la esfera interna de actuación operativa a implementar, parte importante (básica, nos atreveríamos a señalar) es el organigrama de la institución. Este, sea el que sea, debe ser, en todo caso, reflejo de la misión y los valores internos establecidos, pero también de los principios y objetivos definidos.

Sin entrar a definir qué modelo es mejor o peoł ${ }^{7}$, sí nos gustaría recordar que, sea éste el que sea, el mismo debe ser establecido sin olvidar que debe desarrollar (y con fundadas garantías de éxito) las funciones y procesos internos a desarrollar por la ADR. Asimismo, es necesario resaltar que tan importante como el diseño del modelo concreto de organigrama funcional, es la configuración y delimitación de los espacios de decisión y de relación definidos entre las distintas unidades funcionales. Estos espacios permiti rán a la ADR disponer de una serie de estructuras que garanticen en todo momento la toma de decisiones y las relaciones de coordinación entre los distintos componentes.

45 Eurada (1999a): pp. 34-40.

46 Agencia de Innovacion y Desarrollo de Andalucia IDEA (2006), p. 22.

47 A título de ejemplo, y de forma sintética, hemos de señalar que la ADR andaluza apuesta, según su Plan Estratégico 2005/2008, por un nuevo organigrama basado en los siguientes componentes funcionales: un Centro Corporativo (Dirección General, Secretaría General y cuatro Direcciones de Área), los Sectores Estratégicos (unidades, cuatro en 2006, vinculadas a sectores considerados en cada momento como estratégicos), los Entes Instrumentales (especializados en la gestión de servicios de apoyo a la actividad de la Agencia: tres en 2006), y las Gerencias Provinciales (ocho, una por cada provincia andaluza). 
Lógicamente, la definición de estos procesos es otra de las tareas a reati zar por cada ADR. Si bien estos deberían ser explicitados en el denominado 'mapa de procesos' de cada organización, al menos en su conjunto deben responder a la siguiente caracterización tipológica: procesos estratégicos (dirigidos a definir los ámbitos prioritarios en los que debe centrar su inter vención principal la Agencia de Desarrollo); procesos operativos (procesos cuyos resultados son directamente focalizados sobre los 'clientes'); y pro cesos soporte (procesos internos de la organización y tendentes a asegurar el buen funcionamiento de la misma, así como coadyuvar al resto de los procesos definidos). Todo este conjunto de procesos deben conformar el núcleo central de la actividad concreta de cada ADR, desarrollándose estos sobre la base de una serie de áreas de actuación que conforman el armazón orgánico y funcional de las distintas Agencias de Desarrollo ${ }^{48}$.

\subsection{El planteamiento estratégico}

Como último aspecto a tener en cuenta en el proceso de reconfiguración del marco de actuación operativa de las ADR, resulta necesario ocuparse del planteamiento estratégico que debe guiar todo el diseño de actuaciones hasta aquí efectuado. Y es que sin el establecimiento de unas líneas estratégicas que ayuden a definir y orientar toda laactuación de la ADR, lo referido hasta aquí no tendría ningún sentido.

Esta orientación estratégica, al ser parte integrante y necesaria del marco de actuación teórica, aunque por su naturaleza y efectos de alcance integral haya de ser tratada como capítulo aparte, también debe estructurarse en torno a los dos niveles ya conocidos: la perspectiva externa y la interna, que pueden asimilarse a la estrategias de actuación y de organización, respectivamente ${ }^{49}$.

48 Para continuar ejemplificando, hemos de señalar que la Agencia de Innovación y Desarrollo de Andalucía IDEA ha establecido, según su Plan Estratégico 2005/2008, hasta un total de 20 áreas funcionales, que cubren desde los recursos humanos y materiales a los asuntos europeos, pasando por la comunicación, las tareas de innovación, la de emprendedores o la de infraestructuras.

49 Por razones de claridad conceptual y de continuidad con el 'corpus' teórico-práctico que se está presentando, nosotros seguimos utilizando la metodología diseñada específicamen te por la ADR andaluza. No obstante, en EURADA (1999a), pp. 73-97 puede encontrarse una interesante reflexión sobre la programación estratégica regional (concepto, metodologías, medidas y requerimientos) que puede ser útil a la hora de afrontar el diseño 'ad hoc' del 
En cada una de estas esferas han de identificarse los distintos planos o ejes que, a su vez, han de articular tanto las líneas estratégicas como, dentro de éstas, las medidas o actuaciones específicas a desarrollar, para intentar alcanzar el buen éxito del modelo a implementar. Evidentemente, cada ADR en concreto, y en función de la configuración de su marco de actuación operativa, fijará un planteamiento estratégico u otro ${ }^{50}$.

Sea cual sea éste, una parte importante del mismo pasa por afrontar un in eludible cambio en los mismos valores de la organización que, como ejemplo inmediato, pasa por una transformación radical en el mismo lenguaje a utilizar por las ADR. Por todas las unidades funcionales y en todas las áreas existentes.

Y es que habremos de coincidir en que "la evolución de las ideas de la Economía regional viene marcada por un cierto cambio en el lenguaje". ${ }^{51}$

\section{CUADRO 1:}

Hacia una reconfiguracion del lenguaje de las ADR

\begin{tabular}{|c|c|}
\hline ANTIGUO MODELO & NUEVO MODELO \\
\hline Empresa ayudada & Cliente \\
\hline Seguridad en el puesto de trabajo & Desarrollo profesional \\
\hline Centralización & Descentralización \\
\hline Dirección por instrucciones & Dirección por objetivos \\
\hline Ofrecer calidad & Ser calidad \\
\hline Subvencionar & Incentivar \\
\hline Sociedad de la información & Sociedad del conocimiento \\
\hline Dirección vertical & Equipos multidisciplinares \\
\hline
\end{tabular}

Fuente: Instituto dE Fomento dE ANDALUCIA IFA (2004), p. 23.

modelo aunque, algunos aspectos de la misma, puedan ser considerados como desfasados y obsoletos a la luz de los requerimientos pretendidos.

50 Agencia de InNovacion y Desarrollo de Andalucia IDEA (2006), pp. 37-69: el planteamiento es tratégico de la ADR andaluza se concreta en 5 ejes (gestión de la innovación en Andalucía; fomento y extensión de la cultura emprendedora; promoción del desarrollo económico y de la cohesión social y territorial; apuesta por la comunicación y la relación con los agentes del sistema ciencia-tecnología-empresa; y transformación de la ADR andaluza a nivel organiza tivo interno para cumplir con sus nuevas funciones y estrategias), 22 líneas estratégicas y 124 medidas que se desarrollan a través de una serie de acciones recogidas en su catálogo de actuaciones.

51 E. Furió Blasco (1996), p. 19. 
Dentro de la planificación estratégica, tampoco debe olvidarse que la misma, en todo caso, debe responder a los principios y objetivos (tanto ex ternos como internos) marcados y, lo que es más importante, para intentar valorar la eficacia y eficiencia del mismo debe ser capaz de contar con un buen sistema de gestión y con un óptimo plan de seguimiento y evaluación. El primero nos permitirá responder a la cuestión de qué se hace; el segun do, a una cuestión no menos importante: cómo se hace. En este último nos centraremos.

A nuestro juicio, bien podemos afirmar que si de algo ha adolecido tra dicionalmente el modelo español de ADR ha sido de la falta de un buen sistema de seguimiento, hasta el punto de ser éste uno de los aspectos menos valorados y peor considerados en el planteamiento estratégico de las Agencias de Desarrollo. Debido a esta insuficiencia, es preciso acudir a las fuentes de la doctrina comparada ${ }^{52}$, para poder estar en disposición de implementar un óptimo y eficaz sistema de evaluación estratégica. Y es que, a través de este sistema, y mediante la verificación de los resultados obtenidos -los cuales deben ser mensurables y cuantitativos (sin desdeñar, obviamente, los de índole cualitativa, más complejos de medir)- estaremos en condiciones de conocer críticamente la tarea desarrollada por la ADR, de acuerdo al conjunto de indicadores previamente seleccionados, sean cuales sean estos. Como vemos, antes de seleccionar estos, debemos saber qué queremos medir y, lo que es más importante, en función de qué estrategia $\mathrm{y} / \mathrm{o}$ finalidad en concreto. ${ }^{53}$

En cualquier caso, los indicadores seleccionados que deben permitir medir el estado de ejecución de las actuaciones acometidas, deben ser de dos tipos: de resultados (que nos permitan medir los efectos directos, de calidad y rendimiento de las actuaciones individuales, es decir, el grado de cumplimiento de los objetivos específicos) y de impacto (que midan la re percusión de las actuaciones conjuntas en el logro de los objetivos generales del plan diseñado).

52 En especial la escuela inglesa. A este respecto, recomendamos J. KNox ET AL. (2005), donde podemos encontrar un completo (y práctico) desarrollo sobre la implementación metodológica de un marco de evaluación en las ADR. En el contexto español, y centrándose en el caso de Castilla y León, destacamos a B. URUEÑa GuTIÉRREZ (2004).

53 J. KNox ET AL. (2005), p. 2: "The evaluation framework must start from an agreed understanding of what RDAs are intended to achieve"/"El marco de evaluaci ón debe iniciarse desde una comprensión coincidente de lo que las ADR intentan conseguir". 


\section{Resumen y Conclusiones}

En pleno siglo XXI y a la luz de los cambios producidos en los últimos años en la realidad económica, en general, y empresarial, en particular, las Agencias de Desarrollo Regional han de empezar a transformarse necesa riamente para adaptarse a la nueva realidad imperante, si no quieren verse incapacitadas (funcional y estratégicamente) para continuar desarrollando su actuación operativa. Para esto, no es suficiente con introducir cambios tácticos en el modelo, sino que sólo a través de una auténtica reconfigura ción de su marco teórico de actuación estratégica, es posible que las ADR puedan seguir contribuyendo al desarrollo económico y social del ámbito territorial donde operan.

De acuerdo a esta premisa, y una vez identificadas las líneas donde en la actualidad se insertan tanto las nuevas estrategias del desarrollo regional como de la política europea a este respecto, hemos querido ensayar un intento de reconfiguración teórica del marco de actuación de dichas Agencias de Desarrollo Regional, tomado como ejemplo más inmediato el acometido por la ADR andaluza: la Agencia de Innovaci ón y Desarrollo de Andaluc ía IDEA que, como primer ejemplo de las mutaciones y cambios a efectuar, ha sido fruto de la transformación integral del antiguo Instituto de Fomento de Andalucía (IFA), origen y germen de dicha Agencia.

Lógicamente, uno de los motivos -tal vez el m ás importante- que haya podido originar la reflexión estratégica en el seno de la ADR andaluza, bien pudiera venir motivado por la misma constatación de las deficiencias existentes en su anterior modelo operativo. Entre éstas, podrían destacarse la falta de concreción conceptual del anterior modelo que, entre otros proble mas, imposibilita efectuar una valoracón objetiva de la misma actuacón del modelo; el peso creciente de las decisiones deíndole no técnica, que puede conducir a tomas de posicón no siempre defendibles desde el punto de vista de la eficiencia y eficacia económicas; una burocratización progresiva en su actividad y desempe ño; la escasez de ' benchmarking', que impide tomar como referencia ejemplos de buenas prácticas; las disfunciones existentes con otros entes de la misma Administraci ón donde se insertan, los cuales debieran compartir objetivos comunes; la desequilibrada apuesta por los incentivos de tipo financiero como instrumentos de actuacón preferente; la rigidez y obsolescencia de su estructura; etc. Todo esto, evidentemente, ha podido implicar una pérdida de credibilidad en la ADR, anén de producir un claro detrimento en su capacidad de funcionamiento y operatividad. 
Para intentar solucionar, o al menos paliar, estas disfunciones, ha sido pre ciso que la ADR andaluza apostara por una reconfigurámi en profundidad de su modelo. Así, y a través de un repaso a su Plan Estraégico, hemos podido ejemplificar y extraer recomendaciones que hemos pretendido elevar a la categoría de generales, debido -tal y como se ha reflejado- a la congruencia de éstas con los postulados de la nueva estrategia de Desarrollo Regional, en general, y con la nueva Pol ítica Regional Europea, en particular, donde las mismas se insertan. El objetivo de nuestro trabajo s ólo ha intentado encauzar las actuales perspectivas anal íticas en el campo del desarrollo regional con las soluciones operativas a implementar por las Agencias de Desarrollo Regional.

A modo de conclusión final, sirvan nuestras reflexiones para establecer los principios de una nueva guía de actuación donde situar los imprescindibles cambios a efectuar por el conjunto del modelo de Agencias de Desarrollo Re gional. Y es que creemos que el nuevo modelo analítico que aquí ensayamos puede llegar a ser una herramienta muy útil para otras ADR. En posteriores trabajos intentaremos comprobar, a la luz de nuestras recomendaciones, qué distintas vías han seguido las ADR y cuáles han sido, en su caso y si procede, los resultados obtenidos. No obstante, y sea cual sea el camino emprendido por cada una de las ADR, a nuestro juicio lo peor sería que no se acometiera ninguna transformación en el que podemos denominar ya como su modelo tradicional de actuación operativa. Esto podría llegar a invalidarlas como tales Agencias de Desarrollo Regional, ya que al no saber insertarse con éxito en la nueva estrategia del Desarrollo Regional quedan, en la teoría y en la prá $€$ tica, invalidadas operativamente por incumplimiento manifiesto de la misma caracterización funcional y conceptual que las identifica como tales.

\section{Bibliografía seleccionada}

Agencia de Innovacion y Desarrollo de Andalucia IDEA (2006), Plan estratégico 2005/2008, Sevilla, Junta de Andalucía, 79 págs.

ANSELl, Chris (2000), "The networked polity: regional development in Western Europe", Governance: an international journal of policy and administration, Vol. 13, no 3, pp. 303-333.

Aranguren B aena, Francisco Antonio (2005), "Una aproximación al modelo español de Agencias de Desarrollo Regional (ADR)", Revista de Fomento Social, nº 240, pp. 589-623. 
Consejeria de Innovacion, Ciencia y Empresa (2004), Plan de Innovación y Modernización de Andalucía, Sevilla, Junta de Andalucía, 102 págs.

Coronado Guerrero, Daniel (1997), "El proceso de evaluación de las políticas económicas y regionales: una revisión de métodos y experienciasRevista de Estudios Regionales, nº 47, pp. 37-81.

Coronado Guerrero, Daniel et al. (1999), "Innovación tecnológica y desarrollo regional", ICE, n⿳⺈ 781, pp. 103-116.

Dyson, Jane et al. (1998), "Regional Development Agencies Bill (Bill 100 of 1997/98)", Research Paper, 98/7, pp. 26.

Eurada (1999a), Creation, Development and Management of RDAs, Bruxelles, Eurada, 169 págs.

- (1999b),Development Agencies and Enterprises (Working document-dialogue meeting with $D G X X I I I)$, Bruxelles, Eurada, 42 págs.

EUROPEAN COMMISSION (1994), Guide to financial engineering techniques used by the European Commission in the context of regional policy, Luxembourg, Office for official publications of the European Communities, 57 págs.

Fontela Montes, Emilio (2000), El empresario del siglo XXI, Madrid, Pirámide, 136 págs.

Furió Blasco, Elies (1996), Evolución y cambio en la econom ía regional, Barcelona, Ariel, 157 págs.

GuZMÁn Cuevas, Joaquín et al. (2001), "Un modelo explicativo de la emergencia de empresarios", Economía Industrial, nº 340, pp. 151-162.

HALKIER, Henrik et al. (1998), Regional Development Agencies in Europe, London, Jessica Kingsley Publishers Ltd, 376 págs.

HALKIER, Henrik (2000), "Regional policy: an inter-organisational approach", Regional and Industrial Policy Research Paper, nº 37, pp. 53.

HugOnNIER, Bernard (1999), "Regional development tendencies in OECD Coun tries", Keynote presentation to regional Australia summil,arliament House, Camberra, 27-29 October.

Knox, Jim et al. (2005), Evaluating the impact of England's Regional Development Agencies. Developing a methodology and evaluation framework (Final report to the Department of Trade and Industry and the study Steering Group), London, PA Consulting and SQW Ltd, 118 págs. 
Instituto de Fomento de Andalucia IFA (2004), Un nuevo Instituto de Fomento de Andalucía (Libro Blanco), Sevilla, Junta de Andalucía, 58 págs.

MoncAYo J IMÉnez, Edgar (2002), Nuevos enfoques te óricos, evoluci ón de las políticas regionales e impacto territorial de la globalizaci ón, Santiago de Chile, CEPAL (Serie Gestión Pública, nº 27), 78 págs.

OECD (2000), "Local development and job creation", Policy Brief, february 2000, pp. 12.

Palavicin Corona, Eduardo Iván (2000), "Presentación de las nuevas estrategias para el desarrollo regional en la actualidad", Gaceta de Economía, no 10 , pp. $215-232$.

Pigou, Arthur Cecil (1944), Teoría y realidad econ ómica, México, Fondo de Cultura Económica, 148 págs.

RoBERTS, Peter (1999), The new regional agenda in the UK: changing roles, struc tures and functions of Regional Development Agenciqsaper presented at the $39^{\text {th }}$ European Regional Science Association, Dublin, August, 17 págs.

Russo, José Luis (1997), Las disparidades regionales en Argentina y sus efectos sobre los sistemas agroalimentarios en el marco del MERCOSUR. Tesis Doctoral, Córdoba, ETSIAM, 517 págs.

Soler i Marco, Vicente (2000), "Verificación de las hipótesis del distrito indus trial”, Economía Industrial, nº 334, pp. 13-23:

ToRiBıo MuÑoz, M ${ }^{\text {a }}$ del Rosario et al. (2001), "El desarrollo econ ómico y el empresariado andaluz: el caso de C ádiz y Sevilla", Revista de Estudios Regionales, $\mathrm{n}^{\circ}$ 60, pp. 175-205.

URUEÑa GutiÉRREZ, Baudelio et al. (1996), Technological policies and regional development in Spain: the role of Regional Development Agencies , paper presented at the 36 th European Regional Science Association, Zurich, August, 18 págs.

Urueña GutiérRez, Baudelio (2004), Cómo medir la eficiencia de las empresas públicas autonómicas: un estudio de casos con aplicacön a Castilla y León, Valladolid, Universidad de Valladolid, 188 págs.

Villaverde Castro, José(1992), Los desequilibrios regionales en Espãa, Madrid, Instituto de Estudios Económicos, 333 págs. 